Laser Chem., 1999, Vol. 19, pp. 165-168

Reprints available directly from the publisher Photocopying permitted by license only
(C) 1999 OPA (Overseas Publishers Association) N.V. Published by license under the Harwood Academic Publishers imprint, part of The Gordon and Breach Publishing Group.

\title{
TIME-RESOLVED ULTRAVIOLET RESONANCE RAMAN OF PROTEIN STRUCTURAL CHANGES IN THE KL-INTERMEDIATE OF BACTERIORHODOPSIN
}

\author{
SHOJI KAMINAKA and RICHARD A. MATHIES* \\ Department of Chemistry, University of California, Berkeley, CA 94720, USA
}

(Received 7 April 1997)

To obtain high quality time-resolved ultraviolet resonance Raman (UVRR) spectra of transient intermediates in the bacteriorhodopsin (BR) photocycle, we developed a new UVRR spectrometer. A home-made $F=3.5$ prism prefilter was used in front of a $50 \mathrm{~cm}$ CCD detected spectrograph to give high throughput, wide tunability, and excellent stray light rejection along with low dispersion. Using this system, we obtained $239.5 \mathrm{~nm}$ excited time-resolved UVRR spectra of BR which revealed small but significant features associated with the formation of the KL-intermediate at $10 \mathrm{~ns}$ delays. This difference spectrum exhibits intensity decreases at $1624,1561,1012$ and $763 \mathrm{~cm}^{-1}$ due to an altered environment of one or more Trp residues and a frequency shift of the Tyr $\nu_{8 \mathrm{~b}}$ band at $1602 \mathrm{~cm}^{-1}$. These signals show that the photoisomerization of retinal from all-trans to 13-cis induces significant changes in the structure and environment of aromatic residues that line the retinal binding pocket in only $10 \mathrm{~ns}$.

Keywords: Ultraviolet resonance Raman spectroscopy; time-resolved Raman spectroscopy; bacteriorhodopsin; prism prefilter; KL-intermediate; BR photocycle

\section{INTRODUCTION}

Methods have been developed for performing time-resolved UV resonance Raman (UVRR) spectroscopy with the overall goal of

\footnotetext{
*Corresponding author. Tel.: 1-510-642-4192; Fax: 1-510-642-3599; e-mail: rich@) zinc.cchem.berkeley.edu
} 
studying functional protein structural changes with nanosecond time resolution. This paper reports on the development of these spectroscopic techniques and their use to obtain ns time-resolved spectra of the bacteriorhodopsin (BR) photocycle. Early UVRR studies revealed significant protein structural changes upon conversion to the $M$ intermediate $[1,2]$ but the $\mathrm{S} / \mathrm{N}$ was such that any significant protein changes in the KL-intermediate could not be discerned. However, recent ns time-resolved step-scan FTIR spectroscopic studies from our group suggested the possibility of structural changes between the lightadapted form and the KL-intermediate [3]. To obtain higher quality time-resolved UVRR spectra of KL and the other BR intermediates, we therefore developed a new UVRR spectrometer with a prism prefilter that has produced high quality time resolved spectra.

\section{EXPERIMENTAL METHODS}

Our UVRR detection system consists of a home-made tunable $F=3.5$ prism prefilter, a $50 \mathrm{~cm}$ Spex $500 \mathrm{M}$ single monochromator with $1200 \mathrm{gr} / \mathrm{mm} 500 \mathrm{~nm}$ blazed gratings operated in second order, and a UV-sensitive CCD detector (LN/CCD-1100PBUVAR, Princeton Instruments). The prism prefilter consists of a Littrow-type prism (Apex angle: $20^{\circ}$ ) in a Czerny-Turner configuration. This system gives high throughput, tunability and stray light rejection along with low dispersion. As a result, the system can be used for Raman experiments from the visible to the deep ultraviolet $(200 \mathrm{~nm})$, even though a $500 \mathrm{~nm}$ blazed grating is used in the spectrograph stage. A $100 \mu \mathrm{M}$ lightadapted-BR solution at $\mathrm{pH} 7.4$ was recirculated in a thin wall Suprasil quartz capillary cell. The photocycle was initiated with a $5 \mathrm{~ns}$ laser pulse at $532 \mathrm{~nm}$ and probed with a hydrogen shifted Raman pulse at $240 \mathrm{~nm}$. To observe the KL-intermediate, a $10 \mathrm{~ns}$ time delay was achieved using an optical delay line. The prism prefilter was able to completely reject the contamination of $532 \mathrm{~nm}$ stray light and visible fluorescence background in the UVRR spectrum of the KLintermediate. In addition, the Rayleigh scattering from the $239.5 \mathrm{~nm}$ probe beam was strongly rejected. To eliminate any artifacts, we alternately recorded pump-probe and probe-only spectra for 6 minutes 
using the same flow cell set-up and the same BR sample. These spectra were accumulated for a total of 90 minutes.

\section{RESULTS AND DISCUSSION}

The difference spectrum in Figure 1 reveals small but significant features associated with the formation of the KL-intermediate. This difference spectrum exhibits intensity decreases at 1624, 1561, 1012 and $763 \mathrm{~cm}^{-1}$ of one or more trp residues and a frequency upshift of the Tyr $\nu_{8 \mathrm{~b}}$ band at $1602 \mathrm{~cm}^{-1}$. Weaker difference features were also observed in the Trp doublet around $1350 \mathrm{~cm}^{-1}$ and Tyr $\nu_{7}$ mode around $1200 \mathrm{~cm}^{-1}$. These signals show that the photoisomerization of retinal from all-trans to 13-cis induces significant changes in the structure and environment of aromatic residues that line the retinal

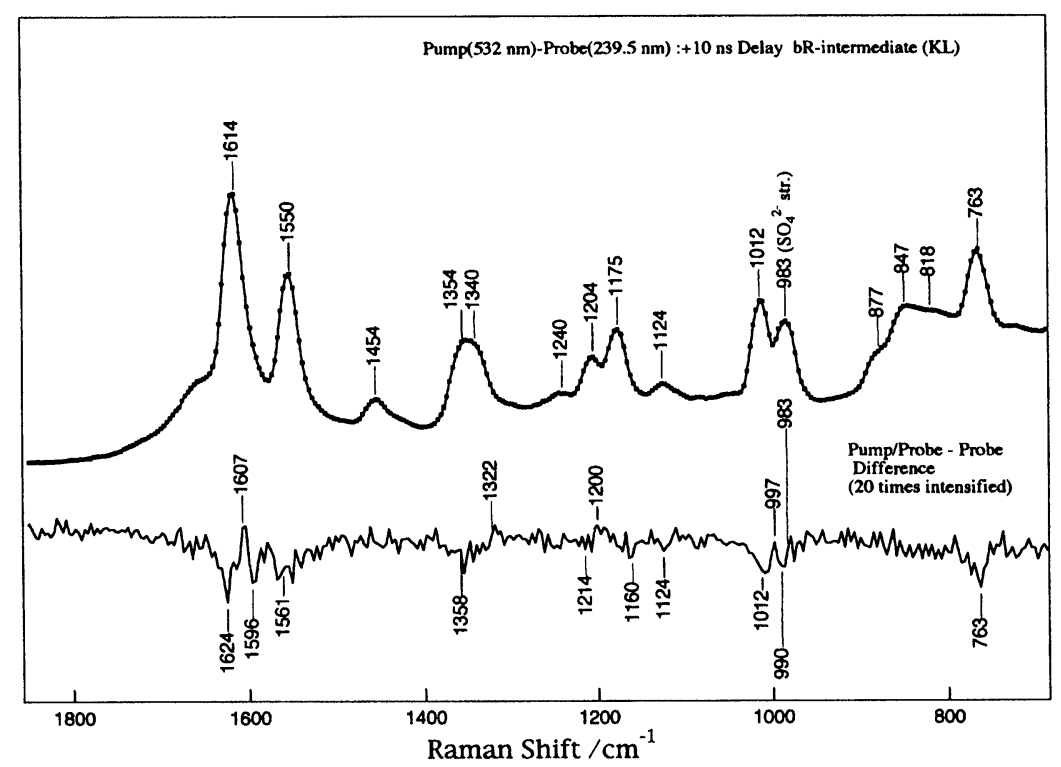

FIGURE $1 \quad 10$ ns time-delayed pump-probe UVRR spectra of bacteriorhodopsin (upper, solid-line), probe-only UVRR spectra of bacteriorhodopsin (upper, dotted-line), and its difference spectrum (lower, pump-probe minus probe-only). The difference spectrum has been blown up by a factor of 20 . All these spectra were not subjected to any mathematical smoothing. 
binding pocket in only $10 \mathrm{~ns}$. Judging from the distances and locations of aromatic amino acids in the chromophore binding site [4], these difference features might be ascribed to Trp86 [5] and Tyr57 [6]. To test these speculations, site specific mutagenesis is now being used to identify the specific residues that are involved.

\section{References}

[1] Ames, J. B., Ros, M., Raap, J., Lugtenburg, J. and Mathies, R. A. (1992). Biochemistry, 31, 5328.

[2] Harada, I., Yamagishi, T., Uchida, K. and Takeuchi, H. (1990). J. Am. Chem. Soc., $112,2443$.

[3] Hage, W., Kim, M., Frei, H. and Mathies, R. A. (1996). J. Phys. Chem., 100, 16026.

[4] Henderson, R., Baldwin, J. M., Ceska, T. A., Zemlin, F., Beckmann, E. and Downing, K. H. (1990). J. Mol. Biol., 213, 899.

[5] Fodor, S. P. A., Pollard, W. T., Gebhard, R., van den Berg, E. M. M., Lugtenburg, J. and Mathies, R. A. (1988). Proc. Natl. Acad. Sci. USA, 85, 2156.

[6] Braiman, M. S., Mogi, T., Stern, L. J., Hackett, N. R., Chao, B. H., Khorana, H. G. and Rothschild, K. J. (1988). Proteins Struct. Funct. Genet., 3, 219. 